\title{
Late Thrombolytic Treatment In A Patient With Ischemic Stroke Caused By Biatrial Thrombus
}

Halit CINARKA ${ }^{1}$, Mevlut KARATAS ${ }^{1}$, Songul OZYURT ${ }^{1}$, Aziz GUMUS ${ }^{1}$, Unal Sahin ${ }^{1}$, Servet KAYHAN ${ }^{*}$

1. Department of chest disease, School of medicine, Recep Tayyip Erdogan University, Rize, Turkey

\begin{abstract}
Venous thromboembolism is a preventable disease when necessary precautions are taken and it occurs along with deep vein thrombosis and pulmonary embolism. Mortality related to venous thromboembolism may be high in the acute phase of the disease and it may become chronic. Intracardiac thrombus may be detected in some venous thromboemboli cases. Cardiac embolism is responsible for most of the ischemic strokes which can be very mortal or may cause serious morbidity when they are not treated in time. In this report, we aimed to present the results of late antithrombolytic treatment in a 77-year old patient who developed deep vein thrombosis, biatrial thrombosis and ischemic stroke following hydrocephalus shunt operation.
\end{abstract}

Key words: Venous thromboembolism; ischemic stroke; thrombolytic therapy

\section{Introduction}

Deep vein thrombosis and pulmonary embolism are two components of venous thromboembolism and they share common risk factors. According to the data from United States of America, it was reported that the risk for developing venous thromboembolism is $1 / 1000$ and incidence increases with increasing age. Pulmonary thromboembolism (PTE) is a preventable disease with high mortality and morbidity, which may recur and diagnosis is sometimes difficult. It generally occurs when thrombus fragments originating from deep veins of lower extremity occlude pulmonary artery and/or its branches. Clinical symptoms may be masked or delayed due to concomitant diseases. Patients with suspected PTE are classified as low, medium and high probability according to their symptoms and risk factors. This classification is useful in empirical diagnosis and treatment approach (1). Intracardiac thrombus sometimes accompanies venous thromboembolism, which may lead to increased ischemic stroke risk when it is not detected early and left untreated. It was shown that about $1 / 5$ of all ischemic strokes originate from cardiac emboli. Unless there is no contraindication, it is very important to initiate

\section{Corresponding author:}

Dr Servet Kayhan

Department of chest disease, School of medicine, Recep Tayyip Erdogan University, Rize, Turkey

Email: kayhanservet@gmail.com Phone and fax: +904642123015

Receive date: 2015-03-05 | Accept date: 2015-04-15| Publish date: 2015-05-03

DOI: 10.7575/aiac.abcmed.15.03.03.09 
thrombolytic treatment after ischemic stroke to decrease mortality and prevent further injury.

\section{Case presentation}

77-year old male patient referred to our pulmonary diseases outpatient clinic from neurosurgery outpatient clinic due to 1 week history of dyspnea, fatigue and cough. His past medical history revealed hydrocephalus shunt operation 2 months ago, Parkinson disease and history of cerebrovascular event. He had a 20 year-pack smoking history and quit smoking 20 years ago. His family history revealed nothing remarkable. On physical examination, his general condition was fair, he was conscious, oriented and cooperated. He had hydrocephalus shunt operation 2 months ago, which resulted in gait and balance disorder and immobility. Vital signs examination revealed heart rate $96 / \mathrm{min}$, respiratory rate $24 / \mathrm{min}$, arterial blood pressure $110 / 60 \mathrm{mmHg}$, temperature $36.8 \mathrm{C}$ and oxygen saturation $89 \%$ on room air. All laboratory parameters were normal except anemia (hemoglobin: $8.75 \mathrm{~g} / \mathrm{dl}$ ). On auscultation, breath sounds were bilaterally coarse and heart sounds were arrhythmic. Electrocardiogram showed atrial fibrillation. On thorax computerized tomography (TCT), main pulmonary artery was patent but its two main branches had filling defects which were consistent with pulmonary thromboemboli. It also revealed cardiomegaly and hypodense lesions in both atriums, which were consistent with thrombus (Figure 1). In addition, lower lobe pulmonary arteries of both lungs had filling defects consistent with pulmonary embolism. The patient was consulted to cardiology department for echocardiography (ECHO) to verify pulmonary embolism. ECHO was reported as (Figure 2); "right atrial mobile echogenic focus consistent with thrombus, which is $3.5 \times 1.3 \mathrm{~cm}$ in size and attaching to septum with its one end, left atrial mobile echogenic focus consistent with thrombus, which is about $5 \mathrm{~cm}$ in length, attaching to interatrial septum with its one end and sometimes prolapsing into left ventricle, biatrial dilatation, pulmonary arterial pressure (PAP) is $50 \mathrm{mmHg}$, ejection fraction is $48 \%$, signs of right ventricular overload, mild aortic valve insufficiency-tricuspid valve insufficiency and global left ventricular hypokinesia were detected". Bilateral lower extremity Doppler ultrasonographic examination was reported as; "No flow is detected in the right main femoral vein and superficial femoral vein, findings are consistent with chronic thrombosis. Right popliteal vein has chronic thrombosis. Left main femoral vein is thin in caliber. It can be compressed. Chronic thrombus detected in the left popliteal vein. Venous drainage occurs via collateral venous structures".

The patient was consulted to cardiology and cardiovascular surgery departments to determine the treatment modality. Cardiovascular department declared that intracardiac thrombus operation risk for this patient was high due to his age and general clinical condition, while cardiology department declared that thrombolytic treatment was not suitable for the patient due to his stable clinical condition. After these consultations, enoxaparin sodium 0.8 IU $2 \times 1$ s.c. treatment was initiated. On the second day of admission, the patient developed change in consciousness and weakness in all extremities. The patient was consulted to neurology department with the suspicion of cerebrovascular event. Neurology department consultation note was as; "Right facial asymmetry, right hemiparesis 4/5 mkm, right Babinski extensor leak". Diffusion magnetic resonance (MR) imaging was reported as; "Diffusion restriction in the wide patchy acute ischemic lesion located in the left posterior temporal region and posterior to sylvian fissure, ventriculomegaly in 3 and both lateral ventricles, widespread and multiple chronic ischemic-gliotic lesions in both centrum semiovale" (Figure 3). It was decided to apply 


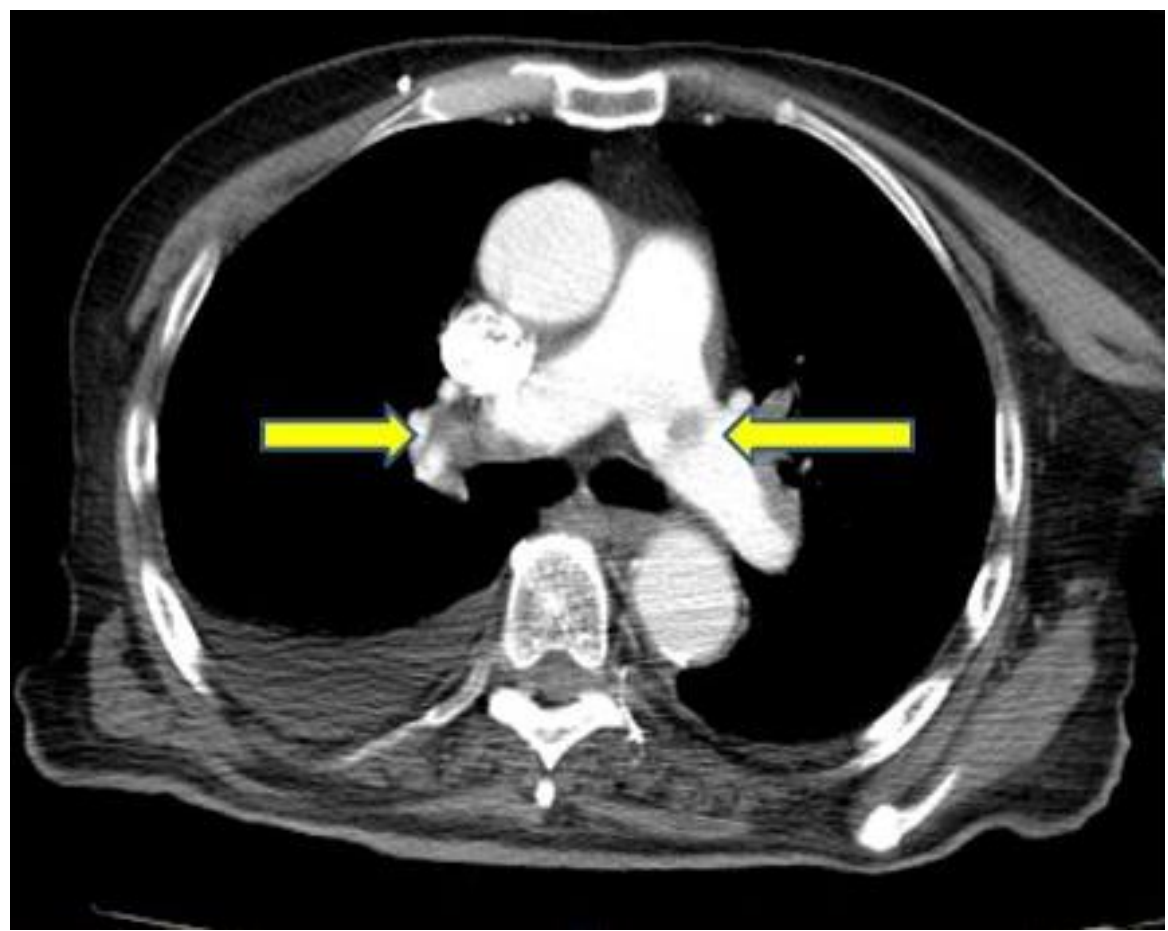

Figure 1: Computed tomographic images of pulmonary embolism in main pulmonary arteries (arrows)

thrombolytic treatment. The patient's relatives were informed about the necessity and the risks of thrombolytic treatment. The patient's relatives gave consent for thrombolytic treatment 6 hours after the initiation of ischemic stroke. Recombinant tissue plasminogen activator reteplase $10 \mathrm{U}$ iv bolus was applied and repeated 30 minutes later as $10 \mathrm{U}$. Although it was decided to apply thrombolytic treatment at the 2 . hour after the initiation of cerebrovascular event, it was applied at 6 . hour due to late consent given by patient's relatives. No complication was observed during and after thrombolytic treatment. Patient's neurological signs related to cerebrovascular event recovered quickly. Oxygen saturation reached to $97 \%$. ECHO performed on the first day after thrombolytic treatment (Figure 4) revealed no thrombus in right and left atriums and pulmonary pressure was measured as $40 \mathrm{mmHg}$. INR was followed up under enoxaparin sodium $0.8 \mathrm{ml} 2 \times 1 \mathrm{sc}$. and oral warfarin treatment. Anticoagulant treatment was resumed with coumadin when
INR dose reached a stable value. Coumadin dose was arranged and patient was discharged to be followed up by outpatient clinic. On the follow up, it was observed that his general condition was completely restored, gait and balance disturbance recovered and the patient gained mobilization. At present, he is on the 12 . month of warfarin treatment and been followed up monthly.

\section{Discussion}

Venous thromboembolism (VTE) is a condition which includes deep vein thrombosis (DVT) and pulmonary thromboembolism (PTE). It may present with sudden death and its diagnosis sometimes may be difficult due to concomitant diseases. In 2004, 317.000 deaths related to VTE were reported in six European countries with a total population of 454.4 million (2). PTE risk increases with increasing age and it is 10 fold higher after the age of 80 when compared to ages of 45-50 (1).

$20 \%$ of all ischemic strokes originate from cardiac embolism (3). Cardiac embolism is rare 


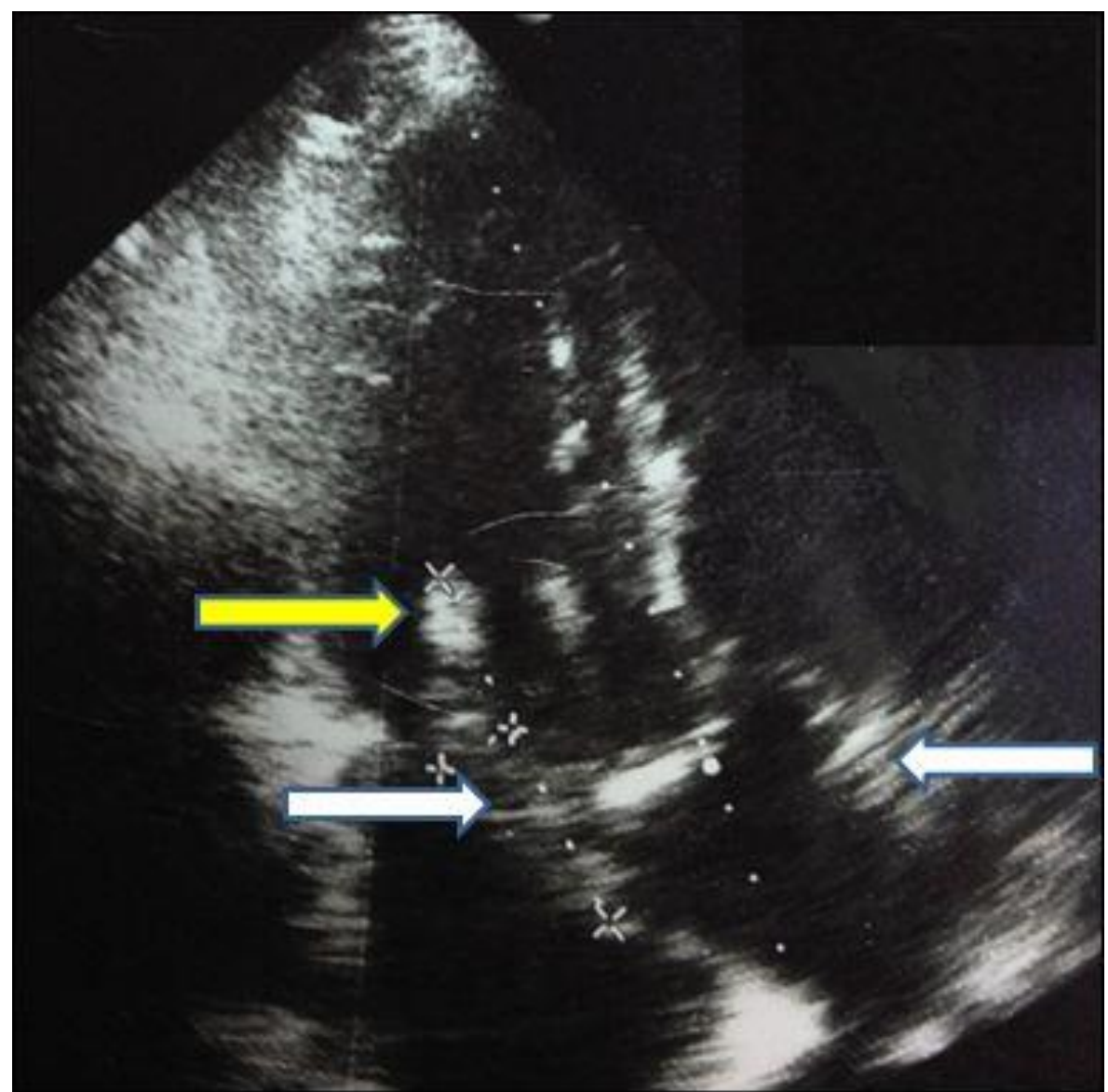

Figure 2: Echocardiographic images of biatrial thrombus (white arrow) and ventricular thrombus (yellow arrow)

when compared to strokes caused by nonembolic mechanisms and causes more extensive brain damage by occluding intracranial arteries with longer diameters. Main causes of cardioembolic stroke include atrial fibrillation (most common), acute myocardial infarction (AMI), ventricular thrombus (20\%), structural heart defects, cardiac tumors $(15 \%)$ and valvular heart diseases $(15 \%) \quad(4,5)$. Atrial fibrillation is the most common cause of cardiac embolism (45\%) and its incidence increases with increasing age. TOAST study focused on acute ischemic stroke and used clinical signs and etiology for classification. According to this classification, causes of acute ischemic stroke are as follows: large artery atherosclerosis, cardioembolism, small vessel occlusion, ischemic stroke related to other determined causes and ischemic stroke of unknown etiology (6).
Treatment of acute ischemic stroke includes thrombolytic agents and mechanical removal of thrombus (7). There is limited data on the risk/benefit ratio of i.v. thrombolytic treatment in ischemic strokes related to cardiac thrombus. In the literature, there are also some cases of cardiac thrombus related ischemic stroke which have been treated with i.v. thrombolytic r-tPA (8). Cardiovascular surgery department did not approve surgical intervention for our case. Nine published randomized studies recommend the use of thrombolytic agents in the treatment of acute ischemic stroke. In our case, after the application of reteplase as thrombolytic treatment, intracardiac thrombus disappeared on ECHO and no thrombus recurrence was seen. The aims of early thrombolytic treatment are to provide early reperfusion of cranial vascularity, minimize the neurological damage

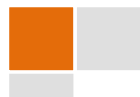




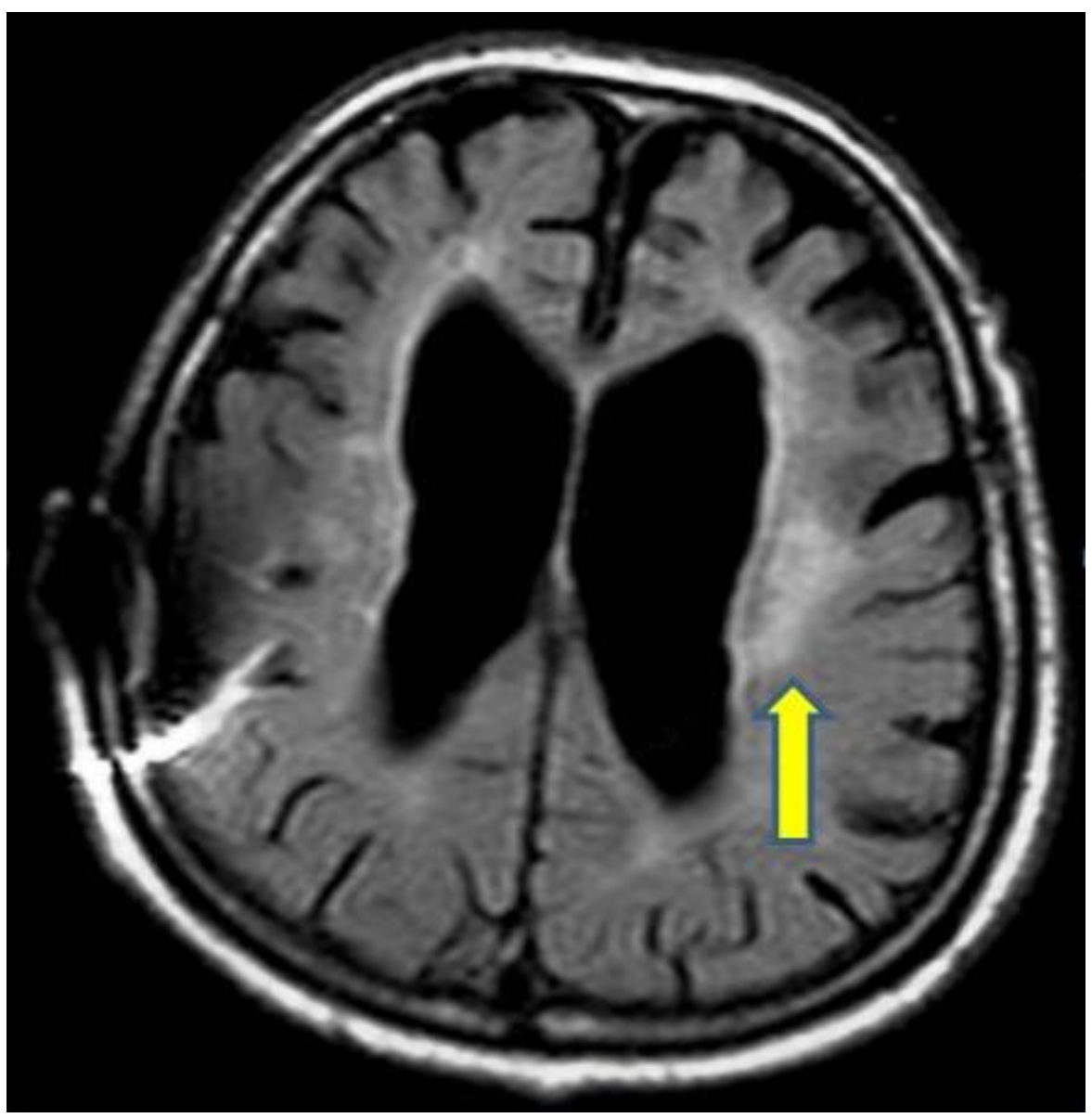

Figure 3: Acute ischemic lesion in the brain (yellow arrow)

and decrease the stroke related mortality. Thrombolytic treatment with intravenous r-tPA provides early vascular reperfusion (7). It has been reported that in acute stroke, initiation of i.v. r-tPA treatment within 3 hours after the beginning of neurological symptoms provides good functional results but has little or no effect on mortality, while initiation of the agent within 3-4.5 hours is less effective when compared to initiation within 3 hours but still provides good clinical results (7). It has also been shown that initiation of the treatment within 4.5-6 hours provides a moderate improvement in the functional results but increases the mortality risk (7). Our case had bilateral DVT, atrial fibrillation, bilateral PTE and biatrial cardiac thrombus. In spite of low molecular weight heparin treatment, he developed acute ischemic stroke on the second day of the treatment. It is probable that acute ischemic stroke resulted from atrial fibrillation and intracardiac thrombus. Initiation of i.v. $r$ tPA treatment was delayed to 6 . hour because of late consent given by patient's relatives. It was observed that neurological symptoms of the patient began to regress within 2 hours. Although the literature data reveals that delayed stroke treatment is a handicap, recovery of pulmonary symptoms after treatment, disappearance of intracardiac thrombus, quick recovery of neurological symptoms and 12 months follow up under warfarin treatment without any morbidity and mortality can be accepted as satisfactory results for our case. 


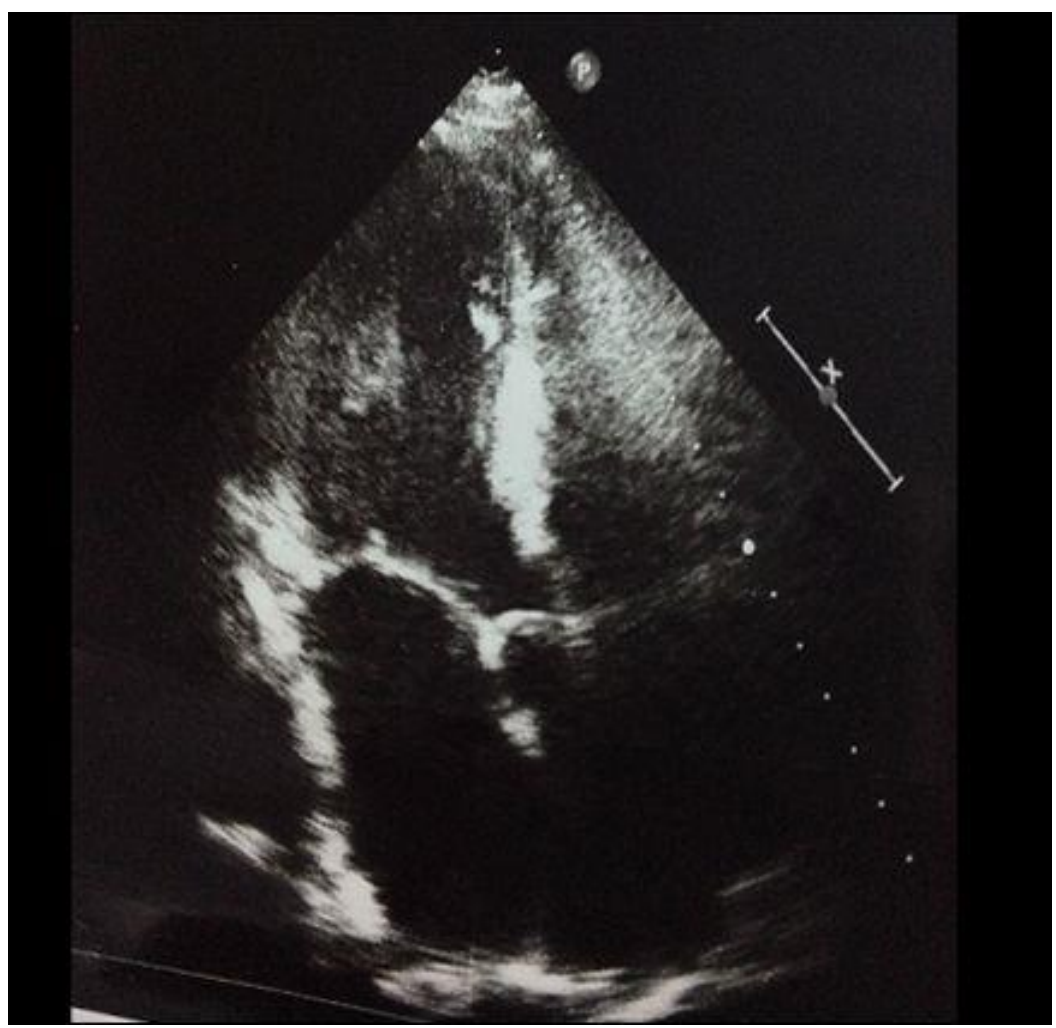

Figure 4: Echocadiograpghic images did not reveal any thrombus after thrombolytic treatment with r-tPA

In conclusion, although 6 hours seems relatively late for the initiation of i.v. thrombolytic treatment in patients with acute ischemic stroke who have deep vein thrombosis, pulmonary emboli, atrial fibrillation and intracardiac thrombus, probability of having successful results in selected cases should not be ignored.

\section{Conflict of interest}

There is no conflict of interest in this study.

\section{References}

1. Turkish Thoracic Society, Diagnosis and treatment guide of pulmonary thromboembolism 2009

2. Cohen AT, Agnelli G, Anderson FA, Arcelus JI, Bergqvist D, Brecht JG, et al. Venous thromboembolism (VTE) in Europe: The number of VTE events and associated morbidity and mortality. Thromb Haemost 2007;98(4):756-764.

3. Asinger RW, Dyken ML, Fisher M. Cardiogenic brain embolism: The second report of the cerebral embolism task force. Arch Neurol 1989;46:727-743.

4. Sila CA. Cardioembolic stroke. In: Noseworthy JH, ed. Neurological therapeutics: principles and practice. New York: Martin Dunitz; 2003:450-457.

5. Ferro JM. Cardioembolic stroke: an update. Lancet Neurol 2003;2:177-188.

6. William David Freeman \& Maria I. Aguilar. Prevention of cardioembolic stroke. Neurotherapeutics 2011;8:488-502.

7. Lansberg MG, O'Donnell MJ, Khatri P, Lang ES, Nguyen-Huynh MN, Schwartz NE, et al. Antithrombotic and thrombolytic therapy for ischemic stroke: Antithrombotic Therapy and Prevention of Thrombosis, 9th ed: American 
College of Chest Physicians Evidence-Based Clinical Practice Guidelines. American College of Chest Physicians. Chest. 2012 Feb;141(2 Suppl):e601S-36S. doi: 10.1378/chest.11-2302.

8. Derex L, Nighoghossian N, Perinetti M, Honnorat J, Trouillas P. Thrombolytic therapy in acute ischemic stroke patients with cardiac thrombus. Neurology 2004;57:2122-5. 